\title{
InteractiveGiotto: An Algorithm for Interactive Orthogonal Graph Drawing*
}

\author{
Stina S. Bridgeman ${ }^{1}$, Jody Fanto ${ }^{1}$, Ashim Garg ${ }^{2}$, Roberto Tamassia ${ }^{1}$, \\ Luca Vismara ${ }^{1}$ \\ 1 Center for Geometric Computing, Department of Computer Science \\ Brown University, USA \\ $\{s s b, j r f, r t, l v\} @ c s$. brown .edu \\ 2 Department of Computer Science \\ State University of New York at Buffalo, USA \\ agargQcs.buffalo.edu
}

\begin{abstract}
We present InTERACTIVEGiotTo, an interactive algorithm for orthogonal graph drawing based on the network flow approach to bend minimization.
\end{abstract}

\section{Introduction}

The last fifteen years have seen an impressive growth of the number of existing graph drawing algorithms. Most of them have been devised with the implicit assumption of being used in "batch mode" and are not well suited for being a component of an interactive system. In fact, the typical use of an interactive system consists of the repetition of the following phases: the user is presented with a drawing of a graph (the current drawing); he/she performs a slight modification (an update) of the drawing, by inserting/deleting some vertices and/or edges; a graph drawing algorithm is executed to obtain another drawing (the new drawing). It is important that the new drawing be as similar as possible to the current one, in order to preserve the mental map the user has of the drawing [3, 7]. Motivated by this type of application, various results on interactive graph drawing algorithms have been recently presented. See, for example, $[1,2,4,6,8,9,13]$.

We focus our attention on interactive orthogonal graph drawing algorithms. We recall that in an orthogonal drawing, each vertex is represented as a rectangle and each edge is represented as a chain of alternating horizontal and vertical segments. Papakostas and Tollis [12] describe four scenarios for interactive orthogonal graph drawing, and present algorithms for two of them. The results of an extensive experimental study comparing the performances of two of those scenarios are reported in [10]. Biedl and Kaufmann [1] present a linear time incremental algorithm for orthogonal drawings, where the vertices are given one at a time and their position cannot be changed after the initial placement.

In this paper, we present INTERACTIVEGIOTTO, an interactive algorithm for orthogonal graph drawing based on the network flow approach to bend mini-

^ Research supported in part by the U.S. Army Research Office under grant DAAH0496-1-0013, by the National Science Foundation under grant CCR-9423847, and by a National Science Foundation Graduate Fellowship. 
mization. Our algorithm does not appear to fit into any of the scenarios described in [12]; in particular, the general shape of the drawing is preserved, as in the Relative-Coordinates scenario, but the coordinates of some vertices and/or edges may change by more than a small constant after each update.

The rest of the paper is organized as follows: Section 2 describes INTERACTIVEGIotTo, Section 3 illustrates the features of InTERACTIVEGrotTo with two examples, and Section 4 outlines plans for future work.

\section{A Brief Description of InteractiveGiotto}

In this section we first describe GIOTTO, then we present the innovative features of INTERACTIVEGIOTTO, and finally we discuss how these features are realized.

GroTTо [16] is a successful general-purpose algorithm for producing orthogonal drawings of graphs, and has been widely used in software visualization systems. It is composed of three steps:

Planarization The input graph is, in general, non-planar, i.e., it cannot be drawn in the plane without edge crossings. In this step the graph is converted into a planar graph by replacing each edge crossing with a fictitious vertex. A heuristic is used to reduce the number of edge crossings.

Orthogonalization In this step, GIOTTO constructs an orthogonal representation of the planarized graph. This representation consists of a description of the bends along each edge and of the angles between consecutive edges around each vertex. In particular, Giotro uses the algorithm described in [15], which produces an orthogonal representation with the minimum number of edge bends by means of a reduction to a minimum cost flow problem.

Compaction In this step an orthogonal drawing is constructed out of the orthogonal representation. The total length of the edges is minimized, again using a reduction to a minimum cost flow problem.

Compared to traditional orthogonal graph drawing algorithms, INTERACTIVEGIOTTO can preserve the following properties of the current drawing during an update:

- the embedding of the graph, i.e., the circular ordering of the edges around each vertex;

- the edge crossings;

- the edge bends and, for $90^{\circ}$ edge bends, also their type (left or right);

- for each vertex, if $R$ is the rectangle representing the vertex, the number of corners of $R$ between any two consecutive edges.

Examples illustrating these properties are presented in Section 3. In addition, our implementation of INTERACTIVEGIOTTO has morphing capabilities that allow the transition from the current drawing to the new one to gradually take place on the screen. This gives the user a better idea of the modifications that are taking place in the drawing.

We now discuss how the previous properties are preserved. INTERACTIVEGiotTo is composed of three steps, similarly to GiotTo: 
Planarization The input to the planarization step is no longer a combinatorial description of a graph, as in GIOTTO, but rather a drawing of a graph. Accordingly, GioTTo's planarizer has been replaced by an algorithm that replaces each edge bend and edge crossing with a fictitious vertex. Edge crossings are detected using a segment intersection algorithm on the set of segments representing the edges of the graph. The embedding and the edge crossings of the current drawing are preserved in this step. In the implementation we make use of LEDA, the Library of Efficient Data Structures and Algorithms [5].

Orthogonalization This step takes the embedded planar graph produced during the planarization and constructs an orthogonal representation preserving the edge bends, the type of the $90^{\circ}$ edge bends, and the number of corners between consecutive edges around each non-fictitious vertex. This goal is achieved through a new algorithm inspired by that described in [14]. The orthogonal representation of the current graph is given by the optimal solution of a minimum cost flow problem in a particular network associated with the current graph. The edge bends, the type of the $90^{\circ}$ edge bends, and the number of corners are preserved by setting a target value for the flow in some arcs of the network and solving the constrained minimum cost flow problem. Note that new bends may be introduced by the algorithm, if needed.

Compaction Same as in Giotro.

\section{Using InteractiveGiotto}

In this section we illustrate the main features of INTERACTIVEGIoTTo through two examples, shown in Figs. 1 and 2.

In the first example, the user incrementally constructs the graph in Fig. 3 of [11]. The initial graph drawn by the user is shown in Fig. 1a using grey vertices and non-orthogonal polylines. InTERACTIVEGiotTo is executed and the new drawing is shown in Fig. 1b using white vertices. Note how all the bends are preserved and how all the vertices, except one, are drawn vertically aligned to preserve the number of corners. The user draws other four vertices and seven edges, and executes InTERACTIVEGIotTo again. Note how the crossing introduced by the user is preserved (see Fig. 1c). After the next update, a new row and a new column are created in the middle and at the bottom of the drawing, respectively, in order to accommodate two new edges (see Fig. 1d). Similar updates are performed in the remaining two steps (see Fig. 1e-1f).

In the second example, we describe some other features of INTERACTIVEGIoTto. This time the initial drawing, shown in Fig. 2a using white vertices, is not empty. In the first step, the user adds one vertex and two edges, each containing one bend. Note how one of the edges drawn by the user crosses three existing edges. In the new drawing, shown in Fig. $2 b$, one more bend is introduced by INTERACTIVEGIOTTO along that edge, in order to preserve the crossings and the number of corners. In the next step, the vertices shown in grey in Fig. $2 b$ are deleted by the user. In Fig. $2 \mathrm{c}$, the user, without having redrawn the graph, 


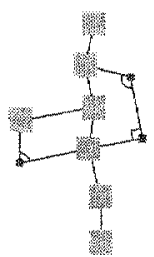

(a)

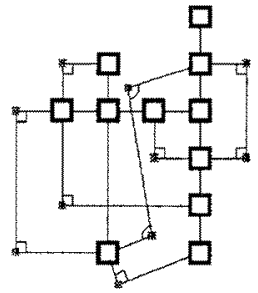

(c)

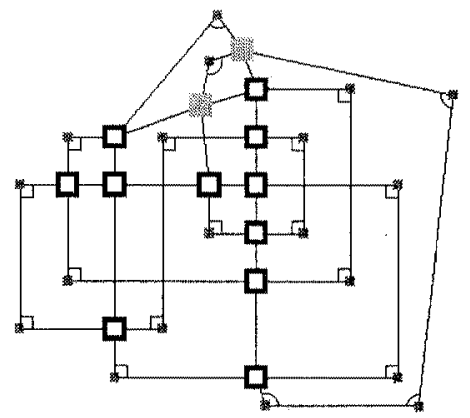

(e)

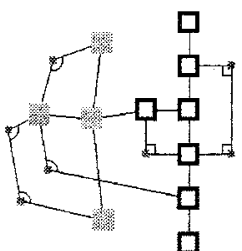

(b)

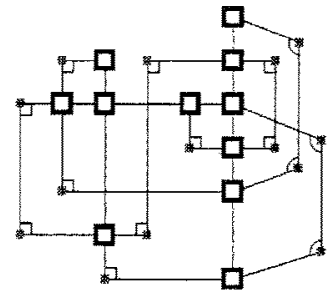

(d)

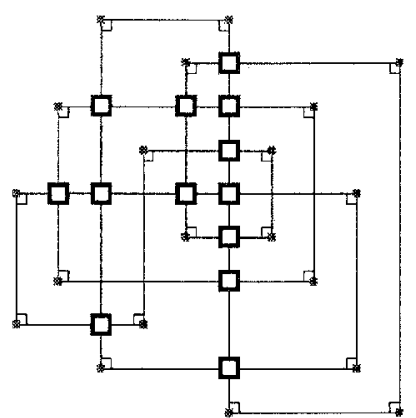

(f)

Fig. 1. Example of use of INTERACTIVEGiotTo: an incremental construction of the graph in Fig. 3 of [11].

moves the subgraph induced by the five vertices show in grey on the outside of the current drawing, reroutes the two existing edges connecting the subgraph to the rest of the graph, and adds one new edge. In the new drawing, shown in Fig. 2d, the shapes of the subgraph and, more importantly, of the rest of the graph are preserved, even though the metric has changed. Note how one of the (non-90 $90^{\circ}$ edge bends introduced by the user is drawn by INTERACTIVEGIOTTO as a $180^{\circ}$ edge bend, i.e., the bend is preserved but its type is not. Finally, the user adds three vertices and nine edges. The drawing produced by INTERACTIVEGIOTTO is shown in Fig. 2e. It should be contrasted with the drawing produced by GiotTo, shown in Fig. 2f. The latter has fewer crossings and bends, a smaller area, and a smaller total edge length, but its shape does not resemble that of the drawing in Fig. $2 \mathrm{~d}$ at all, thus not preserving the mental map the user has of the drawing. 


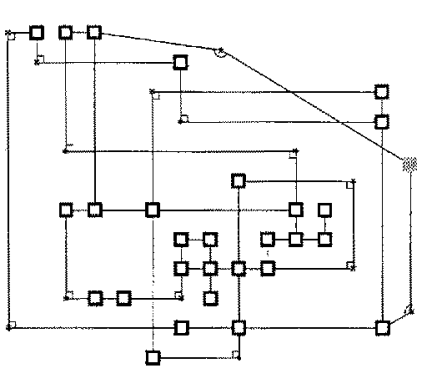

(a)

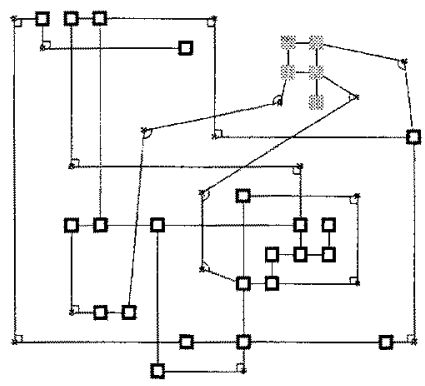

(c)

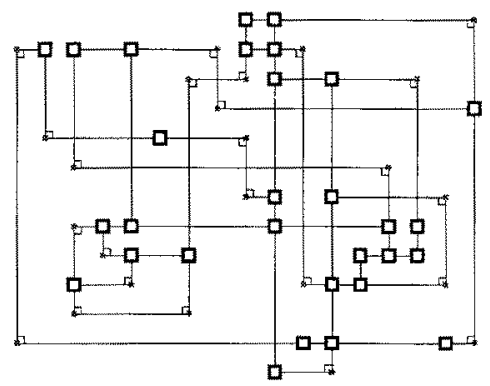

(e)

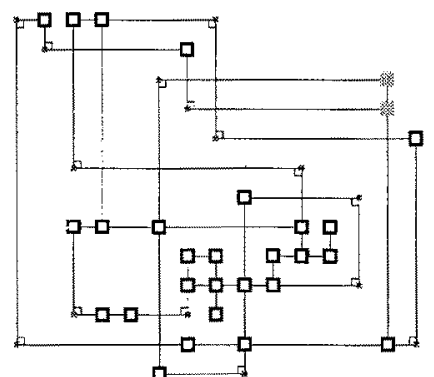

(b)

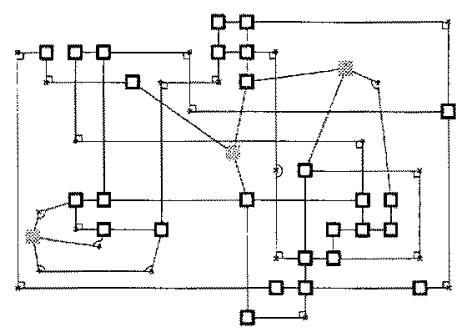

(d)

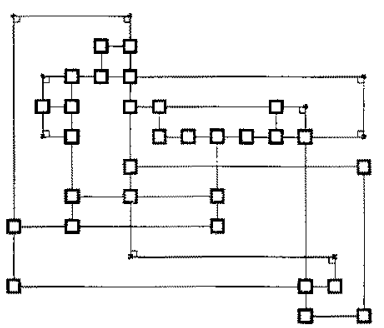

(1)

Fig. 2. Example of use of InteractiveGiotto.

\section{Future Work}

The system presented in this paper is a first prototype. We plan to improve it and expand it in the following directions:

- As in previous works on interactive orthogonal graph drawing, we consider graphs where the vertices have degree at most four. In a future implementation, we will allow arbitrary degree by using a vertex expansion mechanism.

- Currently the edge bends, the type of the $90^{\circ}$ edge bends, and the number of corners are always preserved; this may be too restrictive. In a future implementation, it will be possible to choose whether to favor the preservation of the drawing or the reduction of the number of bends. In particular, the 
user will be able to interactively specify those portions of the drawing whose restructuring is acceptable, and those portions of the drawing whose current shape should be preserved.

\section{References}

1. T. C. Biedl and M. Kaufmann. Area-efficient static and incremental graph darwings. In R. Burkard and G. Woeginger, editors, Algorithms (Proc. ESA '97), volume 1284 of Lecture Notes Comput. Sci., pages 37-52. Springer-Verlag, 1997.

2. R. F. Cohen, G. Di Battista, R. Tamassia, and I. G. Tollis. Dynamic graph drawings: Trees, series-parallel digraphs, and planar ST-digraphs. SIAM J. Comput., 24(5):970-1001, 1995.

3. P. Eades, W. Lai, K. Misue, and K. Sugiyama. Preserving the mental map of a diagram. In Proceedings of Compugraphics 91, pages 24-33, 1991.

4. W. He and K. Marriott. Constrained graph layout. In S. North, editor, Graph Drawing (Proc. GD '96), volume 1190 of Lecture Notes Comput. Sci., pages 217232. Springer-Verlag, 1997.

5. K. Mehlhorn and S. Näher. LEDA: a platform for combinatorial and geometric computing. Commun. ACM, 38:96-102, 1995.

6. K. Miriyala, S. W. Hornick, and R. Tamassia. An incremental approach to aesthetic graph layout. In Proc. Internat. Workshop on Computer-Aided Software Engineering, 1993.

7. K. Misue, P. Eades, W. Lai, and K. Sugiyama. Layout adjustment and the mental map. J. Visual Lang. Comput., 6:183-210, 1995.

8. S. Moen. Drawing dynamic trees. IEEE Softw., 7:21-28, 1990.

9. S. North. Incremental layout in DynaDAG. In F. J. Brandenburg, editor, Graph Drawing (Proc. GD '95), volume 1027 of Lecture Notes Comput. Sci., pages 409 418. Springer-Verlag, 1996.

10. A. Papakostas, J. M. Six, and I. G. Tollis. Experimental and theoretical results in interactive orthogonal graph drawing. In S. North, editor, Graph Drawing (Proc. GD '96), volume 1190 of Lecture Notes Comput. Sci., pages 371-386. SpringerVerlag, 1997.

11. A. Papakostas and I. G. Tollis. Interactive orthogonal graph drawing. Technical report, The University of Texas at Dallas, 1996.

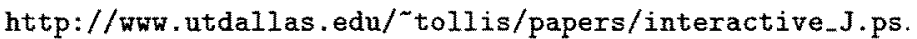

12. A. Papakostas and I. G. Tollis. Issues in interactive orthogonal graph drawing. In F. J. Brandenburg, editor, Graph Drawing (Proc. GD '95), volume 1027 of Lecture Notes Comput. Sci., pages 419-430. Springer-Verlag, 1996.

13. K. Ryall, J. Marks, and S. Shieber. An interactive system for drawing graphs. In S. North, editor, Graph Drawing (Proc. GD '96), volume 1190 of Lecture Notes Comput. Sci., pages 387-394. Springer-Verlag, 1997.

14. R. Tamassia. New layout techniques for entity-relationship diagrams. In Proc. 4 th Internat. Conf. on Entity-Relationship Appraach, pages 304-311, 1985.

15. R. Tamassia. On embedding a graph in the grid with the minimum number of bends. SIAM J. Comput., 16(3):421-444, 1987.

16. R. Tamassia, G. Di Battista, and C. Batini. Automatic graph drawing and readability of diagrams. IEEE Trans. Syst. Man Cybern., SMC-18(1):61-79, 1988. 